\title{
PROPERTIES OF THE SEMI SPIN GLASS GROUND STATE OF GARNETS
}

\author{
A. Koper, A. Lehmann-SzWeykowska, M. Thomas \\ AND R.J. WOJCIECHOWSKI
}

Solid State Theory Division, Institute of Physics, A. Mickiewicz University

Grunwaldzka 6, 60-780 Poznań, Poland

\begin{abstract}
A strong magnetic ordering response to valence-uncompensated doping observed experimentally in yttrium iron garnet, indicates the importance of a thorough analysis of the problem. Within the framework of the classical two-sublattice Heisenberg model the position on the ground state phase diagram, corresponding to the experimentally found values of the superexchange parameters of yttrium iron garnet, turns out to lie close to the semi spin glass region. Thus, in this paper, the magnetic structure factor, the spectrum of magnetic excitations, the density of states and a magnetic contribution to the specific heat, are calculated and discussed.
\end{abstract}

PACS numbers: $75.10 . H k, 75.50 . B b, 81.30 . B x$

Yttrium iron garnet (YIG) belongs to the group of most frequently and thoroughly studied magnetic systems. It is well known but hélas, not quite comprehensible why when doped with valence-uncompensated ions, YIG changes drastically almost all its properties. It seems to be challenging to propose a new model which could explain at least some of the changes. Recently we have concentrated on an analysis of the change in magnetic properties which is displayed by YIG on the valence-uncompensated doping.

As known, pure YIG is an insulating ionic crystal with ferrimagnetic collinear order.

Magnetic properties of the system can be studied with the two-sublattice Heisenberg Hamiltonian [1, 2]

$$
\mathcal{H}=-\frac{1}{2} \sum_{i \neq j} J_{i j}^{a} \boldsymbol{S}_{i} \cdot \boldsymbol{S}_{j}-\frac{1}{2} \sum_{k \neq l} J_{k l}^{d} \boldsymbol{S}_{k} \cdot \boldsymbol{S}_{l}-\sum_{i, k} J_{i k}^{a d} \boldsymbol{S}_{i} \cdot \boldsymbol{S}_{k}
$$

$S$ is the $S=5 / 2$ iron spin operator localized either at the sites of the octahedral $(i, j)$ or tetrahedral $(k, l)$ sublattice. Both the intra- and inter-sublattice coupling constants are limited to nearest neighbours, only, and denoted by $J^{a}, J^{d}$ and $J^{a d}$, respectively.

As already shown before [2], compensating holes produced by the doping, can influence profoundly the superexchange interaction between spins of the $d$ electrons, not destroying, however, the translational symmetry of the system. Thus 
follows importance or even a necessity for obtaining a phase diagram of the magnetic ground state, in terms of varying superexchange parameters.

An analysis of the phase diagram of YIG performed in the classical limit [1] (see the inset in Fig. 1), indicates the occurrence of the semi spin glass phases AF-I and AF-II. Our further discussion will be focused on certain features of these two phases, only. The important observation is that the position $\mathrm{E}$ on the

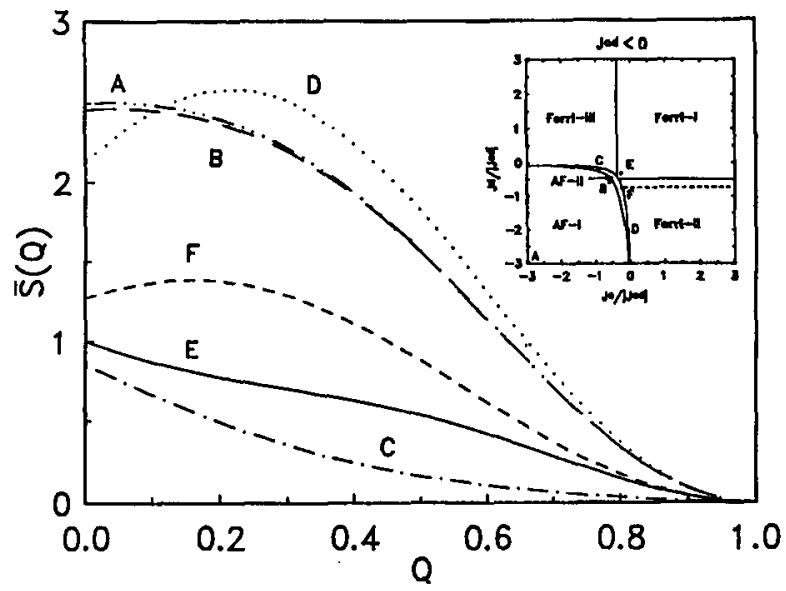

Fig. 1. The mean magnetic structure factor along the $\pi(1,1,1)$ direction for various positions in the phase diagram as indicated in the inset figure (in arbitrary units).

phase diagram corresponding to the experimentally found values of $J^{a}, J^{d}$ and $J^{a d}$ in pure YIG, lies very close to AF-II region. Moreover, as indicated by experimental results, magnetic ordering of YIG can be changed relatively easily by valence-uncompensated doping since the latter strongly influences the superexchange couplings in the crystal. Thus even a small amount of the doping can significantly alter the magnetic state of the system.

In AF-I and AF-II phases, the sublattices $a$ and $d$ show zero resultant magnetic moments. In AF-I phase both sublattices are antiferromagnetically ordered with their moments lying in the same plane. However, the two orderings can be arbitrarily oriented with respect to each other which results in the infinite non-trival degeneracy of the ground state energy or, in other words, an energy valley structure. In AF-II phase the situation is similar but more complex as the two antiferromagnetic structures are arbitrarily oriented with respect to each other in the three-dimensional space. The other regions of the phase plane are described in Ref. [1]. In order to learn more about the magnetic properties of the system in these phases, we examine the magnetic structure factor, the spectrum of magnetic excitations, the magnetic specific heat and the density of states at different but relatively close points of the semi spin glass phase (A, B, C, D, and F as indicated in the inset in Fig. 1). 
The calculations are performed within the framework of the Monte Carlo relaxation method (the same as in Ref. [1]) which is applied to $N_{\mathrm{c}}=40$ spins of an elementary unit cell with the periodic boundary conditions imposed on the superexchange interactions by translational invariance of the Hamiltonian. The quantum fluctuations, however, are neglected in our calculations and the Hamiltonian is replaced by its classical analogon, which actually means that all the spin operators are replaced by the classical vectors $S n_{i}$.

The magnetic static structure factor can be expressed in terms of the Fourier transforms

$$
\boldsymbol{n}(\boldsymbol{q})=\sum_{j=1}^{N_{c}} \exp \left(\mathrm{i} \boldsymbol{q} \cdot \boldsymbol{r}_{j}\right) \boldsymbol{n}_{j}
$$

of the unit vectors $n_{i}$ related to a given equilibrium configuration $v=\left(n_{1}, \ldots, n_{N_{c}}\right)$ :

$$
S_{v}(q)=N_{\mathrm{c}}^{-1} n(q) \cdot n(-q) \text {. }
$$

$\boldsymbol{r}_{1}, \ldots, \boldsymbol{r}_{N_{\mathrm{c}}}$ are positions of the magnetic sites within an elementary unit cell. As seen, the magnetic static structure factor depends explicitly on the configuration $v$. If $\mathcal{V}$ different magnetic configurations (valleys) correspond to the same position in the phase diagram, then the following form of the mean magnetic structure factor:

$$
\bar{S}(q)=\frac{1}{\mathcal{V}} \sum_{v} S_{v}(q)
$$

should be considered.

The knowledge of the magnetic structure factor averaged over the equilibrium configurations of the ground state, enables us to determine a contribution of the antiferromagnetic collinear order to the semi spin glass phases. Our numerical analysis has been performed for wave vectors $q=\pi(1+Q, 1+Q, 1+Q)$, $0 \leq Q \leq 1$, i.e. in the vicinity of the long-range antiferromagnetic order modulation vector. As seen in Fig. 1, no curve is "peaked out" strictly at the value of $\pi(1,1,1)$ corresponding to the antiferromagnetic collinear order, which means that the contribution of the latter to either semi spin glass phase is small.

The frequencies of the harmonic magnons $E_{\alpha}(q), \alpha=1, \ldots, N_{c}$, (cf. [3, 4]) are calculated at the selected positions of the phase diagram. We have found [4] that at each of the latter there occurs at least one acoustic branch. At the position $\mathrm{C}$ and D of AF-II phase, the acoustic branches are only weakly separated from the optical excitations. It is noteworthy that inside the AF-II region, the excitation spectrum is strongly dependent upon a position of the equilibrium spin configuration. An overall dispersion of the most optical lines is not significant, which indicates a rather strong localization of the excitations.

The conclusion is that the range of application of the linear spin wave theory in the semi spin glass phases is reduced to much lower temperatures than it is in the ferrimagnetic phase (position $\mathrm{E}$ ). The result is further supported by the behaviour of the magnetic specific heat [4].

Global characteristics of the spin excitations is given by the density of states (DOS) defined as

$$
g_{v}(E)=\frac{v_{a}}{(2 \pi)^{3} N_{\mathrm{c}}} \sum_{\alpha=1}^{N_{c}} \int_{\mathrm{BZ}} \mathrm{d}^{3} q \delta\left(E-E_{\alpha}(q)\right),
$$


where $v_{a}$ is the volume of an elementary unit cell. The DOS histograms obtained at the five different positions of the phase diagram, are gathered in Fig. 2. In AF-II phase, DOS turns out to be considerably differentiated according to the equilibrium spin configuration $\boldsymbol{v}$.

As follows from a comparison of the discussed physical quantities found for randomly chosen ground state energy valleys, those attributed to a given position in the semi spin glass phases are homogeneous. Moreover, our analysis points out

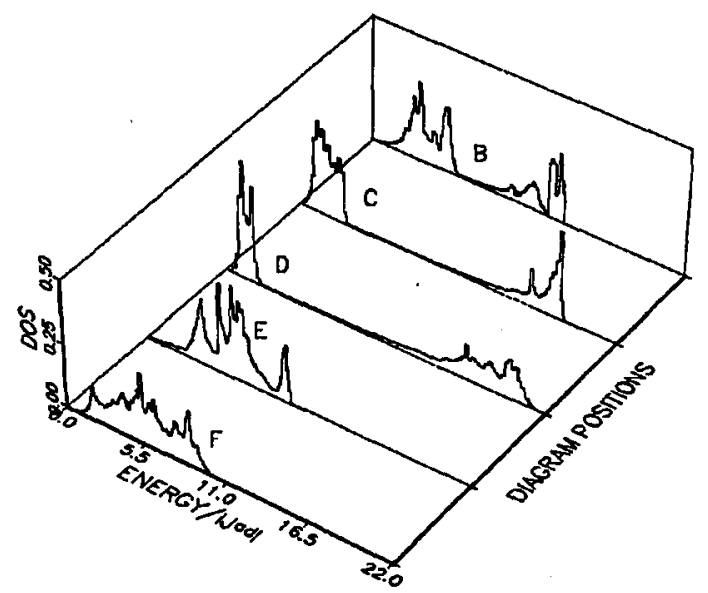

Fig. 2. The density of states (DOS) at five different positions of the phase diagram.

that values of the quantities vary considerably with the position in the semi spin glass phases. This observation sustains the indication that the garnet can be very sensitive even to a small amount of the doping ions.

The research was supported by the Committee for Scientific Research under the grant No. 3 P408 00104.

\section{References}

[1] A. Lehmann-Szweykowska, A. Koper, R.J. Wojciechowski, T. Tomalak, J. Magn. Magn. Mater. 104-107, 447 (1992); A. Koper, A. Lehmann-Szweykowska, R.J. Wojciechowski, Physica B 180-181, 133 (1992).

[2] A. Lehmann-Szweykowska, R.J. Wojciechowski, L. Pust, P.E. Wigen, S. Batra, Phys. Rev. B 43, 3500 (1991).

[3] L.R. Walker, R.E. Walstedt, Phys. Rev. Lett. 38, 514 (1977); Phys. Rev. B 22, 3816 (1980).

[4] A. Koper, A. Lehmann-Szweykowska, M. Thomas, R.J. Wojciechowski, to be published. 